\title{
MICROONDAS EM SÍNTESE ORGÂNICA
}

\author{
Antonio Manzolillo Sanseverino \\ Departamento de Química Orgânica, Instituto de Química, Universidade Federal do Rio de Janeiro, 21949-900 Rio de Janeiro - RJ
}

Recebido em 11/7/01; aceito em 17/10/01

\begin{abstract}
MICROWAVES IN ORGANIC SYNTHESIS. The application of microwave heating to organic synthesis is presented in a concise manner. Issues such as the history of the microwave oven, dielectric heating, reactions techniques (dry reactions, MORE chemistry), domestic ovens, microwave reactors, microwave effect and control of selectivities are discussed. Selected examples from the literature showed faster reactions, improved yields, less thermal degradations and cleaner reactions.
\end{abstract}

Keywords: microwave heating; organic synthesis; green chemistry.

\section{INTRODUÇÃO}

A utilização do forno de microondas de cozinha no preparo ou aquecimento de alimentos é um fato comum nos dias de hoje. O uso de microondas em química analítica já é conhecido desde a década de 70, sendo que entre as aplicações mais importantes podemos citar: a digestão de amostras para análise elementar, a extração de diversas substâncias e a desorção térmica de vários compostos ${ }^{1}$. O aquecimento por microondas também é largamente utilizado em escala comercial na preparação e secagem de alimentos ${ }^{2}$.

Uma aplicação recente é a obtenção de produtos orgânicos em escala de laboratório usando o aquecimento por microondas, onde as reações são conduzidas em forno de microondas de cozinha ou em reatores especificamente desenhados para esta finalidade. Embora esta tecnologia seja parte do nosso cotidiano, como ela surgiu? Quem inventou o forno de microondas? Porque esta técnica despertou um interesse tão grande na área de síntese orgânica?

Portanto, o objetivo deste trabalho é apresentar uma breve introdução sobre a utilização do aquecimento de microondas em síntese orgânica.

\section{O QUE SÃO MICROONDAS?}

As microondas são radiação eletromagnética não ionizante, que possuem uma frequiência que vai de 300 a $300.000 \mathrm{MHz}$ e que corresponde a comprimentos de onda de $1 \mathrm{~mm}$ a $1 \mathrm{~m}$. A região de microondas situa-se entre a região de infravermelho e ondas de rádio no espectro eletromagnético ${ }^{1}$ (Figura 1).

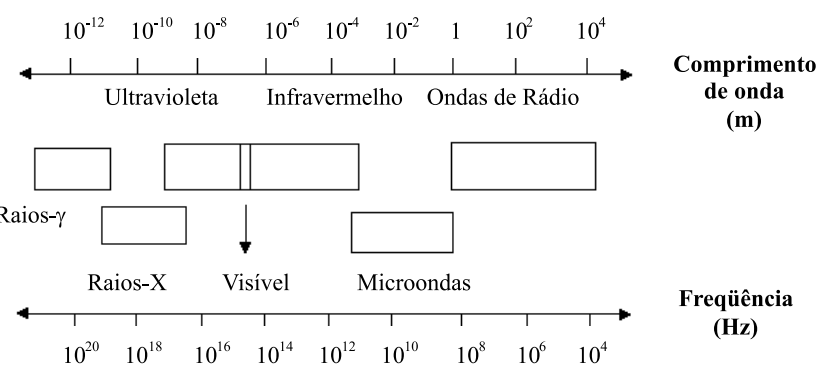

Figura 1. Localização da região de microondas no espectro eletromagnético

\footnotetext{
*e-mail: amsansev@ig.com.br

\# endereço atual: Universidade do Rio de Janeiro (UNIRIO), Escola de Ciências Biológicas, Av. Pasteur, 458, Urca, 22290-240 Rio de Janeiro - RJ
}

\section{A INVENÇÃO DO FORNO DE MICROONDAS}

Cientistas britânicos durante a $2^{\underline{a}}$ Guerra Mundial desenvolveram um dispositivo que gerava microondas chamado magnétron e que era o coração do RADAR ("Radio Detection And Ranging") usado para detectar aeronaves inimigas. O sistema funciona da seguinte forma: o objeto a ser detectado reflete o sinal emitido (as microondas) e o sistema de RADAR detecta o eco deste sinal, e com isto é possível saber a posição, forma do objeto, velocidade e direção de seu movimento.

Havia uma necessidade urgente na Inglaterra de produzir o magnétron em grande quantidade e os cientistas britânicos entraram em contato com os Estados Unidos, de forma que pudessem usar o parque industrial americano para produzir este aparelho que era crucial na defesa da Inglaterra contra os ataques aéreos da Alemanha ${ }^{3}$. Após uma sugestão do laboratório de Radiação do M.I.T. (Massachusetts Institute of Technology), ocorreu um encontro de cientistas britânicos com um engenheiro chamado Percy L. Spencer (1894-1970) de uma pequena companhia americana chamada Raytheon. Spencer, considerou o método de produção do magnétron usado pelos cientistas britânicos como inadequado e pouco prático, tendo informado este fato a estes cientistas. Após isto, ele teria convencido os cientistas britânicos a levar o magnétron para sua casa (um importantíssimo segredo militar da Inglaterra), onde em um fim de semana ele fez diversas mudanças radicais neste aparelho, que não apenas melhoram o processo de fabricação como tornaram o dispositivo mais eficiente ${ }^{3}$. Ao final da Guerra, a Raytheon foi responsável por cerca de $80 \%$ de todos os magnétrons produzidos, e pulou de apenas 15 funcionários para mais de 5.000 (e ainda hoje é uma gigante nos Estados Unidos). Um fato impressionante, era que Spencer possuía apenas o ensino primário, mas era autodidata e na sua época foi considerado como um dos maiores especialistas no campo da eletrônica, tendo 225 patentes em seu nome ${ }^{3}$.

E o forno de microondas, como foi inventado? Os cientistas que trabalhavam com o magnétron já sabiam que além da emissão de microondas também havia geração de calor, mas foi Spencer que percebeu que poderia-se usar radiação eletromagnética para aquecer alimentos. Em 1945, Spencer notou que uma barra de um doce em seu bolso começou a derreter quando ele ficou em frente a um tubo de magnétron que estava ligado, e intrigado por este fato ele conduziu alguns experimentos simples, como preparar pipoca espalhando alguns grãos de milho em frente ao tubo ${ }^{3}$. Em outro, um ovo cru explodiu como resultado do forte aquecimento interno. Pouco tempo depois, no ano seguinte, a Raytheon solicitou a primeira patente 
sobre a utilização de microondas para o aquecimento de alimentos. Em 1947, a Raytheon apresentou o primeiro forno de microondas chamado "Radarange". Este microondas pesava cerca de $340 \mathrm{Kg}$ e possuía cerca de $1,5 \mathrm{~m}$ de altura! O preço era entre US\$2.000 e 3.000 e era vendido para estabelecimentos comerciais. Além disso, este microondas também precisava de refrigeração interna. No final da década de 60 começaram a ser comercializados com sucesso fornos de microondas domésticos (com as dimensões dos atuais e sem necessidade de refrigeração) com preços de US\$ 500. O forno de microondas doméstico tornou-se popular em escala mundial nas décadas de 70 e $80^{3}$. A frequiência de operação destes fornos é de 2,45 $\mathrm{GHz}^{1}$.

\section{O AQUECIMENTO POR MICROONDAS}

O aquecimento por microondas é completamente diferente daquele que ocorre em um forno de cozinha convencional (seja a gás ou elétrico), onde o aquecimento de alimentos ocorre por condução, irradiação e convecção. $\mathrm{O}$ aquecimento por microondas é também chamado de aquecimento dielétrico, e existem dois mecanismos principais para a transformação de energia eletromagnética em calor ${ }^{4}$. O primeiro deles é chamado rotação de dipolo, e relaciona-se com o alinhamento das moléculas (que tem dipolos permanentes ou induzidos) com o campo elétrico aplicado. Quando o campo é removido as moléculas voltam a um estado desordenado, e a energia que foi absorvida para esta orientação nestes dipolos é dissipada na forma de calor. Como o campo elétrico na frequiência de $2,45 \mathrm{GHz}$ oscila (muda de sinal) 4,9 × $10^{9}$ vezes por segundo, ocorre um pronto aquecimento destas moléculas. Uma representação esquemática é mostrada na Figura 2, onde usou-se a água como exemplo.

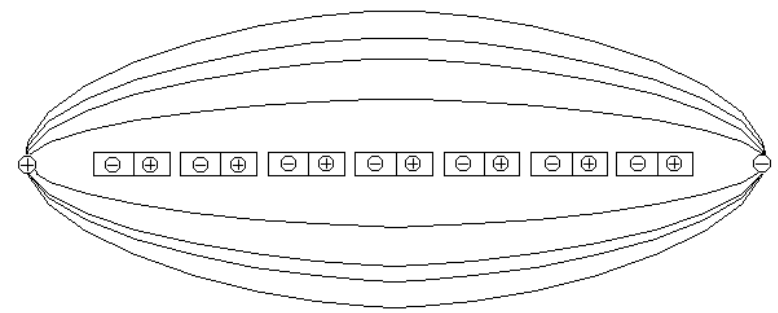

1- Dipolos alinhados com o campo eletromagnético.

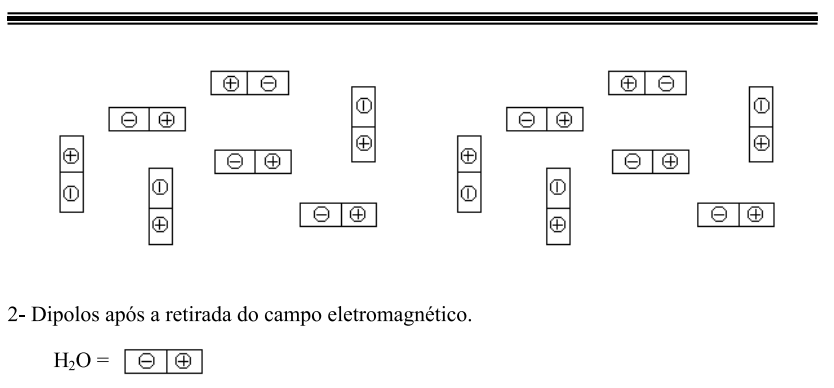

Figura 2. Moléculas de água com e sem a influência do campo elétrico

O segundo mecanismo é chamado de condução iônica, e o calor é gerado através de perdas por fricção, que acontecem através da migração de íons dissolvidos quando sob a ação de um campo eletromagnético. Estas perdas dependem do tamanho, carga, condutividade dos íons dissolvidos e interação destes últimos com o solvente.

O fator de perda dielétrica $\left(\varepsilon^{\prime \prime}\right)$ mede a eficiência da conversão de energia eletromagnética em calor. A constante dielétrica $\left(\varepsilon^{\prime}\right)$ da substância é uma medida que indica a sua polaridade. Já a razão $\varepsilon^{\prime \prime} / \varepsilon^{\prime}$, é numericamente igual a $\tan \delta\left(\tan \delta=\varepsilon^{\prime \prime} / \varepsilon^{\prime}\right)$, sendo chamada de fator de dissipação, que significa a habilidade de uma amostra converter radiação eletromagnética em calor (quanto maior este valor mais a substância é aquecida por microondas). A Tabela 1 mostra várias substâncias e estes parâmetros mencionados ${ }^{1,4}$.

Tabela 1. Constante dielétrica, fator de perda dielétrica e fator de dissipação de algumas substâncias $\left(25^{\circ} \mathrm{C}\right.$ e $\left.3 \mathrm{GHz}\right)$

\begin{tabular}{|c|c|c|c|}
\hline Material & $\varepsilon^{\prime}$ & $\varepsilon^{\prime \prime}$ & $\operatorname{Tan} \delta \times 10^{4}$ \\
\hline GELO & 3,2 & 0,00288 & 9 \\
\hline ÁGUA $\left(25^{\circ} \mathrm{C}\right)$ & 76,7 & 12,0419 & 1570 \\
\hline $\mathrm{NaCl}$ aq. $0,1 \mathrm{M}$ & 75,5 & 18,12 & 2400 \\
\hline $\mathrm{NaCl}$ aq. $0,5 \mathrm{M}$ & 67,0 & 41,875 & 6250 \\
\hline $\mathrm{PrOH}$ & 3,7 & 2,479 & 6700 \\
\hline ETILENOGLICOL & 12,0 & 12 & 10000 \\
\hline HEPTANO & 1,9 & 0,00019 & 1 \\
\hline $\mathrm{CCl}_{4}$ & 2,2 & 0,00088 & 4 \\
\hline
\end{tabular}

Conforme esperado, o gelo praticamente não sofre aquecimento por microondas, pois é uma substância cristalina e ordenada $(\tan \delta \mathrm{x}$ $10^{4}=9$ ). Já a água, é prontamente aquecida por microondas, pelo mecanismo de rotação de dipolo. Soluções aquosas de $\mathrm{NaCl}$ (ou de outro eletrólito) sofrem os dois mecanismos de aquecimento, e são mais aquecidas do que a água pura. A concentração da solução também é importante, como mostra a Tabela 1 para o aquecimento por microondas de soluções de $\mathrm{NaCl}$ de concentrações diferentes, onde uma maior concentração de $\mathrm{NaCl}$ leva a um maior aquecimento. Outras substâncias polares como etilenoglicol e propanol também são prontamente aquecidas por microondas. $\mathrm{O} \mathrm{CCl}_{4}$ possui momento de dipolo nulo e não sofre aquecimento por microondas. $\mathrm{O}$ hexano também não é aquecido por microondas, já que é apolar. No entanto, não é apenas a polaridade da molécula que deve ser levada em consideração, como fica evidente na Tabela 1 para o caso do 1-propanol, que embora possua uma constante dielétrica bem menor que a água, é bem mais aquecido por microondas. O maior aquecimento do 1-propanol quando comparado com a água é explicado pela sua menor capacidade calorífica à $25^{\circ} \mathrm{C}: 2,45 \mathrm{~J} / \mathrm{g} \mathrm{K}$ (o valor para água é 4,18 J/g K) .

Em geral, substâncias polares absorvem bem microondas (como água, acetonitrila, etanol), enquanto que substâncias menos polares (hidrocarbonetos alifáticos ou aromáticos) ou substâncias com momento de dipolo nulo (como $\mathrm{CCl}_{4}, \mathrm{CO}_{2}$ ) absorvem microondas fracamente. Materiais cristalinos altamente ordenados também são pouco aquecidos por microondas (já que não há pouca ou nenhuma rotação de dipolo). Substâncias como teflon e vidro pyrex são transparentes às microondas. Os metais refletem microondas ${ }^{1,4,5}$.

Um exemplo interessante é o caso das porcelanas que possuem $\varepsilon^{\prime}=6,0-8,0$, sendo este valor semelhante para o ácido acético $\left(\varepsilon^{\prime}=\right.$ $6,15)$, mas enquanto o último é prontamente aquecido por microondas, as porcelanas não são, pois trata-se de uma material cristalino e rígido 5 .

\section{MICROONDAS EM SÍNTESE ORGÂNICA}

Os primeiros relatos de reações orgânicas conduzidas em forno de microondas doméstico surgiram em 1986 em dois trabalhos independentes de Gedye ${ }^{6}$ e Guigere ${ }^{7}$. As reações foram conduzidas em frasco selado com uma comparação com o aquecimento convencional (Esquema 1). Uma notável redução no tempo de reação foi observada, embora não tenha havido um controle de pressão. Os primeiros relatos de reações conduzidas em forno de microondas do- 
méstico também levaram aos primeiros relatos de acidentes, pois alguns frascos deformaram ou explodiram devido as condições de irradiação ${ }^{6}$. Apesar disso, inúmeros trabalhos se seguiram após estes relatos iniciais, sendo relatado na literatura diversas reações orgânicas conduzidas com sucesso em forno de microondas doméstico sem modificação, e com segurança segundo os autores destes traba$\operatorname{lhos}^{5,8,9,10,11}$. A utilização de reatores, digestores e fornos modificados também foi investigada após algum tempo ${ }^{12,13}$. A simbologia usada nas reações é: $\mu \nu, \mathrm{MO}$ (microondas) e IMO (irradiação por microondas), também sendo comum indicar a potência na equação química no lugar destes símbolos.
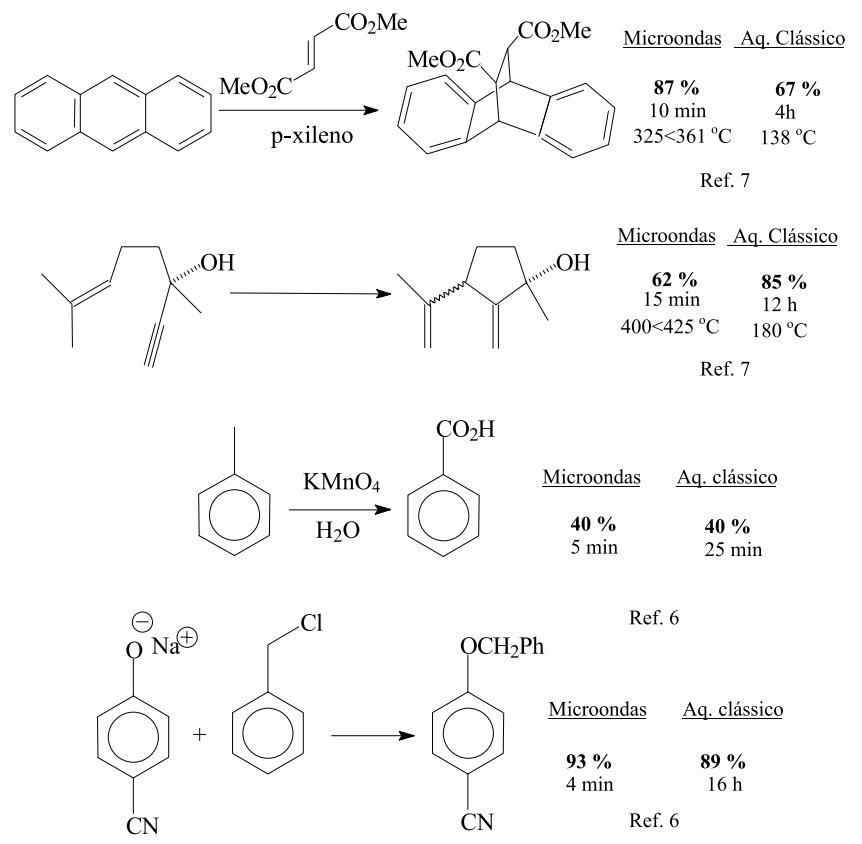

Esquema 1. Primeiras reações orgânicas conduzidas em forno doméstico

As principais vantagens da utilização de energia de microondas sobre o aquecimento convencional ${ }^{14,15}$ (manta, bico de bunsen, placa de aquecimento, etc.) para uma reação química, seriam: a) as taxas de aquecimento em uma reação onde alguma substância (solvente ou um reagente) presente absorva bem microondas são muito maiores que no aquecimento convencional; b) o reator ou recipiente da reação pode ser transparente às microondas (como teflon, por exemplo), a energia é absorvida somente pelos reagentes ou solvente (ou até seletivamente por apenas um dos constituintes da reação); c) a energia é transferida diretamente para a amostra, não havendo contato físico com a fonte de aquecimento; d) a possibilidade de maiores rendimentos, maior seletividade e menor decomposição térmica, vantagens bem documentadas na literatura para várias reações orgânicas em sistemas homogêneos e heterogêneos.

\section{REAÇÕES EM AUSÊNCIA DE SOLVENTE}

Esta técnica tem sido apontada como um procedimento de Química Verde ou Química Limpa, já que não é usado um solvente na reação, o que eliminaria a necessidade de reciclar, descartar e manipular um solvente orgânico (que muitas vezes é tóxico, inflamável e/ ou ataca a camada de ozônio $)^{11,16}$. As reações conduzidas por esta metodologia mostraram grande vantagem em termos de rendimento, tempo e menor formação de subprodutos, onde num experimento típico utiliza-se um gral com pistilo no qual misturam-se os reagentes líquidos com um catalisador sólido (ou suporte, como sílica gel, alumina, argilas e diversos metais impregnados nestes materiais ${ }^{11}$ ) ou misturam-se os reagentes sólidos e irradia-se com microondas.

O rearranjo de pinacol é uma reação bem conhecida na Química Orgânica, e foi conduzido em ausência de solvente em forno de microondas de cozinha usando uma argila (montimorilonita) com íons de Cobre(II) suportados ${ }^{17}$ (Esquema 2). O procedimento com aquecimento convencional levou a menor rendimento e a um tempo muito maior, como mostrado na comparação com o aquecimento convencional (15h!). Outro exemplo, é uma alquilação aniônica conduzida em alumina, que também apresentou grande vantagem em relação ao aquecimento convencional, já que o éster foi obtido em apenas 2 minutos contra um tempo de 5 h no processo convencional $^{18}$ (Esquema 2).

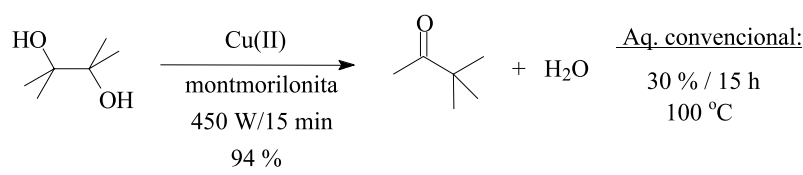

$\begin{array}{cc}\mathrm{CH}_{3} \mathrm{COOK}+\mathrm{C}_{8} \mathrm{H}_{17} \mathrm{Br} \underset{600 \mathrm{~W} / 2 \min }{\stackrel{\mathrm{Al}_{2} \mathrm{O}_{3}}{\longrightarrow}} \stackrel{\text { Aq. convencional: }}{\stackrel{\text { W } \%}{91 \%}} \mathrm{CH}_{3} \mathrm{COOC}_{8} \mathrm{H}_{18}+\mathrm{KBr} & 93 \% / 5 \mathrm{~h} \\ 100{ }^{\circ} \mathrm{C}\end{array}$

Esquema 2. Reações em ausência de solvente com aquecimento dielétrico

Algumas reações tipo Wittig (Wittig-Horner) já foram conduzidas em forno de microondas de cozinha em um frasco aberto, na ausência de solvente e sem preocupação com umidade, pois usou-se um ilídeo estável nas condições experimentais ${ }^{19}$ (Esquema 3). Um fato marcante observado neste trabalho, é que a reação de um derivado de esteróide não ocorreu usando o procedimento tradicional (reação em solução com refluxo e aquecimento convencional $)^{19}$. Na realidade, muitas reações que não ocorrem ou levam a baixo rendimento usando o aquecimento convencional (manta, bico de bunsen, placa de aquecimento, etc.) com solvente ou em ausência de solvente já foram conduzidas com sucesso por aquecimento dielétrico.
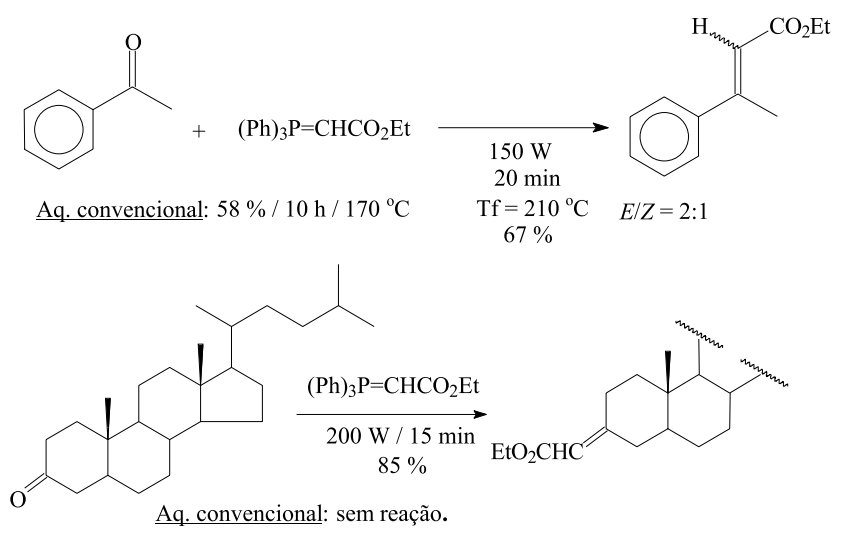

Esquema 3. Reação tipo Wittig em forno de microondas de cozinha

As reações de deslocamento direto $\left(\mathrm{S}_{\mathrm{N}} 2\right.$ - Substituição Nucleofílica de Segunda ordem) também foram conduzidas com sucesso com aquecimento por microondas em ausência de solvente orgânico, como mostra o exemplo da Equação 1 e Tabela $2^{20}$. Neste caso, o nucleófilo (íon fenóxido) é gerado in situ e reage com a epicloridrina levando ao epóxi-éter em rendimentos bons a moderados e tempo reacional de $1 \mathrm{~min}$. Para fenol $(\mathrm{R}=\mathrm{H})$, o aquecimento convencional levou ao mesmo rendimento, porém com um procedimento que durou $6 \mathrm{~h}$. 


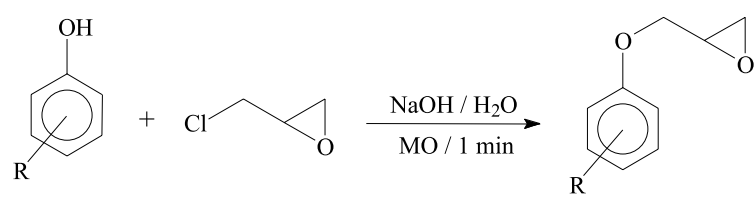

Equação 1. Exemplo de uma reação $S_{N} 2$ com microondas

Tabela 2. Resultados da Reação da Equação 1

\begin{tabular}{cc}
\hline Substituinte: $\mathrm{R}$ & Rendimento $(\%)$ \\
\hline $\mathrm{H}$ & 88 \\
$2-\mathrm{Me}$ & 83 \\
$2-\mathrm{Cl}$ & 88 \\
$4-\mathrm{COMe}$ & 63 \\
\hline
\end{tabular}

A adição de Michael é uma forma importante de formação da ligação carbono-carbono em Química Orgânica, e também foi estudada em forno de microondas doméstico (Esquema 4). O experimental é simples, e consiste em misturar os reagentes líquidos na alumina e proceder a irradiação. De acordo com os autores, o método é especialmente útil para aceptores de Michael cíclicos, tendo vantagem sobre uma metodologia anterior que também usava alumina em ausência de solvente, mas sem o uso de microondas ${ }^{21}$.<smiles>CCOC(=O)CC(C)=O</smiles><smiles>O=C1C=CCCC1</smiles><smiles>CCOC(=O)C(C(C)=O)C1CCC(C(=O)O)C(=O)C1</smiles><smiles>CCOC(=O)CC(C)=O</smiles><smiles>CCOC(=O)C(C(C)=O)C1CCC(=O)C1</smiles>

Esquema 4. Adição de Michael em alumina com microondas

Um demonstração visual do efeito do aquecimento por microondas em reações de polimerização em ausência de solvente já foi relatada em Química Nova ${ }^{22}$, usando monômeros de interesse comercial.

\section{REAÇÕES COM SOLVENTE}

Diversas reações com solventes já foram realizadas com sucesso em forno de microondas doméstico não modificado. A técnica foi desenvolvida principalmente por Bose e colaboradores ${ }^{23}$, e consiste em utilizar solventes polares (portanto que são bem aquecidos por microondas) e de alto ponto de ebulição como DMF (dimetil-formamida, $\left.160{ }^{\circ} \mathrm{C}, \varepsilon^{\prime}=36,7\right)$; etilenoglicol $\left(196-198{ }^{\circ} \mathrm{C}\right) ; \mathrm{PhCl}$ (cloro-benzeno, $\left.131^{\circ} \mathrm{C}\right)$; diglime $\left(\mathrm{CH}_{3} \mathrm{OCH}_{2} \mathrm{CH}_{2} \mathrm{OCH}_{2} \mathrm{CH}_{2} \mathrm{OCH}_{3}, 162^{\circ} \mathrm{C}\right)$. As reações foram conduzidas em um frasco aberto cujo volume é bem maior que o volume da amostra, sendo que o solvente escolhido deve ter um ponto de ebulição cerca de $30{ }^{\circ} \mathrm{C}$ acima da temperatura da reação. Uma revisão sobre esta técnica já foi publicada, possuindo maior riqueza de detalhes sobre estes procedimentos experimentais e com exem- plos de várias reações (algumas com finalidade educacional) ${ }^{10}$. Em alguns casos, um recipiente com água é colocado no microondas para absorver parte da energia, prevenindo um aquecimento muito elevado quando se trabalha com pequenas quantidades de amostra. A técnica é conhecida na literatura como "MORE Chemistry" ("Microwave Oven Reaction Enhancement") $)^{10,23}$.

A preparação de lactamas via adição in situ de ceteno (ciclo adição térmica 2+2) foi uma reação conduzida com irradiação por microondas em forno doméstico com solvente (Esquema 5), onde a respectiva lactama foi obtido em um tempo reacional de apenas 5 minutos e usando um frasco aberto ${ }^{23}$. Como estes compostos tem importância em síntese, principalmente na área farmacêutica, o método é atraente. A reação de Diels-Alder também já foi conduzida por esta técnica ${ }^{23}$, com obtenção de bons rendimentos e curtos tempos de reação (Esquema 5), segundo Bose esta técnica de realizar uma reação de DielsAlder é mais segura do que as reações relatadas com frasco selado ${ }^{6}$, conforme mostrado anteriormente no Esquema 1.
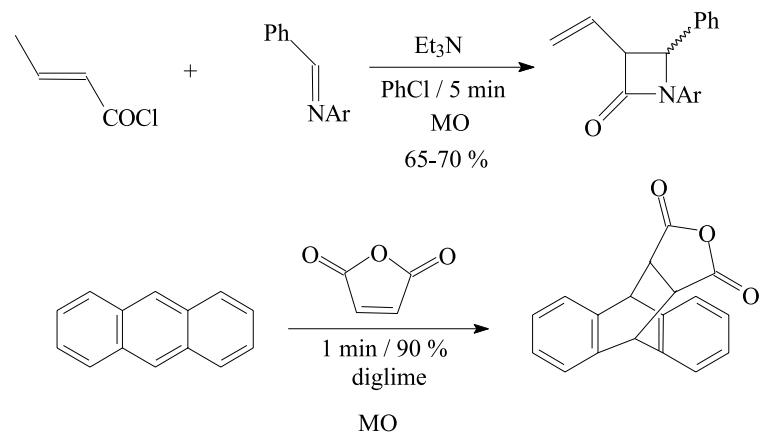

Esquema 5. Algumas reações realizadas com solvente e microondas

A hidrogenação por transferência catalítica ("catalytic transfer hydrogenation") é uma forma simples de realizar uma hidrogenação catalítica sem usar gás hidrogênio, pela reação de um catalisador com um doador de hidrogênio como ácido fórmico, formiato de amônio, hidrazina, e outros ${ }^{24}$. Em geral, a reação é conduzida à pressão atmosférica e com um condensador de refluxo, usando-se etanol como solvente. Assim, é possível realizar uma hidrogenação de vários grupos funcionais em condições suaves (sem necessidade de alta temperatura ou pressão) e sem a necessidade de hidrogenadores. Bose e colaboradores, adaptaram esta técnica para realizar reduções em um forno de microondas doméstico não modificado, usando formiato de amônio como doador de hidrogênio e etilenoglicol como solvente $\mathrm{e}^{23,25,26}$.

O procedimento, de acordo com os autores, foi conduzido sem nenhum problema ${ }^{23,25,26}$, embora a presença de um metal em um forno de microondas doméstico e uma reação de hidrogenação seja surpreendente (e Pd/C é inflamável). Várias funcionalidades foram testadas neste método, e NiRa (Níquel-Raney) ${ }^{25}$ mostrou ser um catalisador mais seletivo do que $\mathrm{Pd} / \mathrm{C}^{23}$, na redução da ligação dupla carbono-carbono sem reação de cisão anelar em certas lactamas (Esquema 6). A redução de ligação dupla carbono-nitrogênio também foi investigada com este método, pela redução de hidrazonas ${ }^{26}$ (Esquema 6).

\section{REATORES DE MICROONDAS}

Reações orgânicas com aquecimento por microondas podem ser conduzidas em microondas modificados ${ }^{27}$, em digestores de microondas (que tradicionalmente são usados em Química Analítica) e em 


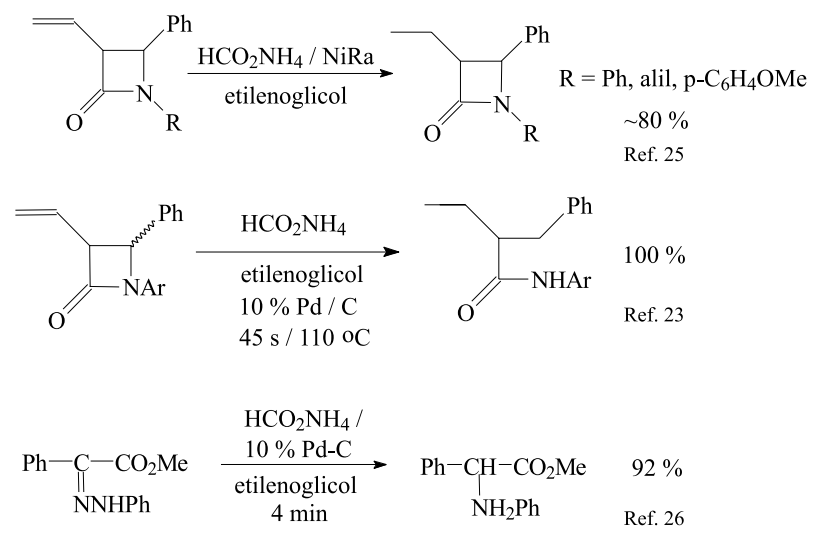

Esquema 6. Reações de hidrogenação em forno de microondas

reatores de microondas ${ }^{12,13}$. A modificação de um forno de microondas para realização de reações com refluxo já foi publicada com detalhes em um artigo da Química Nova, onde uma grande vantagem apontada pelos autores seria o baixo custo destas modificações quando comparado com o preço de um equipamento para laboratório (U\$ $15.000)^{27 c}$. Outros autores também já relataram modificações em forno de microondas com possibilidade de controle de temperatura e pressão, e de realização de refluxo $27 \mathrm{a}, 27 \mathrm{~b}$.

O surgimento de reatores de microondas para condução de reações orgânicas ocorreu como consequiência direta do estudo das reações em forno doméstico, que demonstrou o grande potencial desta técnica. Estes aparelhos, segundo alguns autores, teriam vantagens marcantes sobre o forno doméstico de microondas, tais como: possibilidade de realização de refluxo, utilização de pressão, controle de temperatura e pressão (que não é possível no forno de cozinha) ${ }^{12,13}$. A segurança operacional também seria muito maior, já que o forno de microondas de cozinha não foi fabricado para o uso em Quími$\mathrm{ca}^{12,13}$. Outro fator importante, é que esses reatores operam de forma diferente que um forno doméstico, onde a distribuição de microondas no interior do forno não é homogênea (forno multimodo). Já neste reatores, a distribuição é homogênea (monomodo). Além disso, a potência em um forno de microondas não é, na realidade, possível de variação, pois o que ocorre é uma interrupção seqüencial da irradiação que corresponde aos níveis de potência do seletor (a amostra está submetida sempre a mesma potência, mas por períodos de tempo diferentes). Assim, estes reatores são mais eficientes do ponto de vista energético, e garantiriam uma maior reprodutibilidade de experimentos ${ }^{12,13}$. Outro fato apontado, é a possibilidade de trabalhar com potências menores nestes reatores (15-300 W), que evita decomposição térmica em certas reações conduzidas no forno de cozinha $^{12,13}$.

Alguns exemplos de reações conduzidas em reatores de microondas são mostrados no Esquema $7^{12,28}$.

A reação de síntese do ácido $\alpha$-iodo-acético, segundo Trainor e colaboradores $^{28}$, é conduzida no reator de microondas em batelada num tempo bem menor do que no processo convencional. O segundo exemplo, mostra a obtenção de um éter metílico em $75 \%$ de rendimento, enquanto que a reação com aquecimento convencional (e base no meio reacional) levou ao estireno como produto principal e uma quantidade mínima do derivado de éter metílico ${ }^{28}$. Já a reação do glicerol com acetona e catálise pelo ácido $p$-tolueno-sulfônico, foi conduzida em processo contínuo e levou a $84 \%$ de rendimento do produto em dois minutos de operação, e a comparação foi realizada com a metodologia tradicional que para levar a um rendimento semelhante necessita de um dia (operação em batelada) ${ }^{12}$.

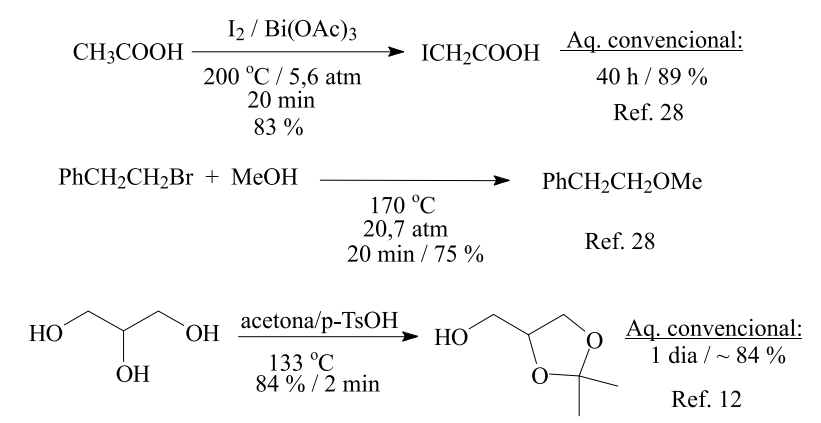

Esquema 7. Algumas reações em reator de microondas

Comparações entre reatores de microondas com aquecimento convencional e/ou aquecimento por forno doméstico foram relatadas por Loupy e colaboradores ${ }^{13}$, em uma revisão sobre o uso de reator monomodo com a técnica de reações em ausência de solvente. A isomerização do eugenol ao isoeugenol (que é utilizado na Química de perfumes e flavorizantes) foi conduzida em ausência de solvente com uma base em presença de um agente de transferência de fase, sendo obtido um rendimento superior no reator monomodo (Equação 2, Tabela 3). Este fato foi explicado pelos autores como sendo resultado da menor potência de irradiação do reator de microondas quando comparada com a do microondas doméstico, o que evitou a decomposição térmica do isoeugenol ${ }^{13}$. O catalisador de transferência de fase aliquat 336 é um sal de amônio quaternário, com predominância de $\mathrm{CH}_{3} \mathrm{~N}\left[\left(\mathrm{CH}_{2}\right)_{7} \mathrm{CH}_{3}\right]_{3} \mathrm{Cl}$ em sua composição.

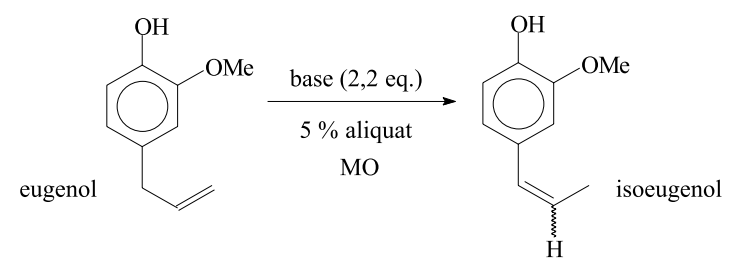

Equação 2. Isomerização do eugenol em ausência de solvente

Tabela 3. Resultados da isomerização do eugenol em ausência de solvente

\begin{tabular}{|c|c|c|c|c|c|}
\hline Base & Aparelho & $\begin{array}{l}\text { Potência } \\
\text { (W) }\end{array}$ & $\begin{array}{c}\text { Tempo } \\
(\mathrm{min})\end{array}$ & $\begin{array}{l}\text { Temp. } \\
\left({ }^{\circ} \mathrm{C}\right)\end{array}$ & $\begin{array}{c}\text { Rendimento } \\
(\%)^{1}\end{array}$ \\
\hline$\overline{\mathrm{KOH}}$ & Reator monomodo & 45 & 12 & 198 & 88 \\
\hline $\mathrm{KOH}$ & $\begin{array}{l}\text { Microondas } \\
\text { doméstico }\end{array}$ & 600 & 3 & 194 & 65 \\
\hline$t \mathrm{BuOK}$ & Reator monomodo & 45 & 18 & 192 & 94 \\
\hline
\end{tabular}

1- Rendimento do isoeugenol. Em todos os casos as conversões são praticamente completas.

Um outro exemplo interessante foi a saponificação de vários ésteres $^{29}$, conduzida em ausência de solvente com catálise por transferência de fase (novamente usou-se aliquat 336) e hidróxido de potássio. $\mathrm{O}$ procedimento conduzido em um digestor de microondas (reator monomodo) mostrou-se superior ao aquecimento convencional (conduzido em banho de areia) e ao aquecimento em forno de microondas de cozinha, como mostram alguns casos selecionados deste trabalho (Esquema 8 e Tabela 4). $\mathrm{O}$ resultado para $\mathrm{R}=\mathrm{nC}_{8} \mathrm{H}_{17}$ é o mais impressionante deste estudo, já que com aquecimento convencional não houve formação do ácido correspondente (e recuperou-se $100 \%$ da matéria-prima) ${ }^{29}$. 


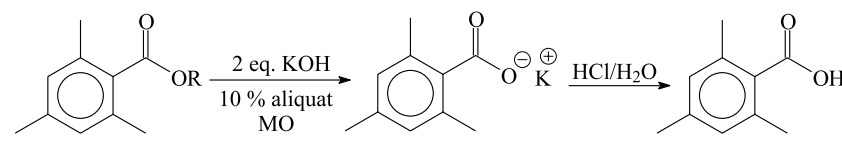

Esquema 8. Saponificação de ésteres por microondas

Tabela 4. Resultados da saponificação de ésteres

\begin{tabular}{|c|c|c|c|c|c|}
\hline Base & Aparelho & $\begin{array}{c}\text { Potência } \\
\text { (W) }\end{array}$ & $\begin{array}{c}\text { Tempo } \\
\text { (min) }\end{array}$ & $\begin{array}{c}\text { Temp. } \\
\left({ }^{\circ} \mathrm{C}\right)\end{array}$ & $\begin{array}{l}(\%) \\
\text { Ácido }\end{array}$ \\
\hline \multirow[t]{3}{*}{$\mathrm{Me}$} & $\begin{array}{l}\text { Microondas } \\
\text { doméstico }\end{array}$ & 250 & 2 & $?^{1}$ & 75 \\
\hline & Reator monomodo & 90 & 2 & 240 & 87 \\
\hline & $\begin{array}{l}\text { Aquecimento } \\
\text { convencional }\end{array}$ & - & 2 & 240 & 38 \\
\hline \multirow[t]{3}{*}{$\mathrm{nC}_{8} \mathrm{H}_{17}$} & $\begin{array}{l}\text { Microondas } \\
\text { doméstico }\end{array}$ & 250 & 2 & $?^{1}$ & 57 \\
\hline & Reator monomodo & 90 & 4 & 223 & 82 \\
\hline & $\begin{array}{l}\text { Aquecimento } \\
\text { convencional }\end{array}$ & - & 4 & 223 & 0 \\
\hline
\end{tabular}

1- Temperatura desconhecida.

Um estudo muito interessante sobre reações orgânicas em água superaquecida com microondas já foi publicado ${ }^{30}$, sendo esta técnica extremamente interessante do ponto de vista ambiental pois utiliza um solvente não tóxico, não inflamável e de baixo custo. A água no estado superaquecido possui propriedades anômalas, que tem despertado a atenção de muitos pesquisadore ${ }^{16,31}$. O aumento da temperatura ambiente até o ponto crítico $\left(\mathrm{T}_{\mathrm{c}}=374{ }^{\circ} \mathrm{C}\right)$ gera um decréscimo substancial na constante dielétrica, ao mesmo tempo em que há um aumento de até 3 ordens de magnitude no produto iônico (Tabela 5) ${ }^{31}$. Este último fato diminui (ou até mesmo elimina) a necessidade de adicionar a uma determinada reação um ácido ou uma base, já que a concentração de íons hidrônio e hidróxido é muito maior do que à temperatura ambiente. A solubilidade de compostos apolares também é bem maior na água superaquecida. Outra razão para usar esta tecnologia é a separação dos produtos da mistura reacional, que é uma etapa fundamental na indústria química (dependendo do processo esta etapa pode alcançar de 60 a $80 \%$ do custo operacional $)^{31}$. Assim, resfriando-se o sistema e abaixando-se a pressão, os produtos são separados da água por uma simples decantação. A utilização de água como solvente para reações orgâni$\operatorname{cas}^{16,32}$ tem aumentado consideravelmente nos últimos anos, sendo que uma revisão sobre o assunto foi, recentemente, aceita para publicação na Química Nova ${ }^{32}$.

A reação da $S(-)$-carvona conduzida com aquecimento em um reator de microondas em batelada leva à formação do carvacrol em ex-

Tabela 5. A água na região supercrítica e próxima a supercrítica

\begin{tabular}{lccc}
\hline Propriedade & Ambiente & $\begin{array}{c}\text { Próxima a } \\
\text { Supercrítica }\end{array}$ & Supercrítica \\
\hline Temperatura $\left({ }^{\circ} \mathrm{C}\right)$ & 25 & 275 & 400 \\
Pressão $(\text { bar })^{1}$ & 1 & 60 & 230 \\
Densidade $(\mathrm{g} / \mathrm{mL})$ & 1 & 0,7 & 0,1 \\
Constante dielétrica & 80 & 20 & 2 \\
Ct. de Ionização & 1 & 1000 & $<0,01$ \\
relativa ${ }^{2}\left(25^{\circ} \mathrm{C}, \mathrm{K}_{\mathrm{w}} \mathrm{K}_{\mathrm{w}}\right)$ & & & \\
\hline
\end{tabular}

1- 1 bar $=1,013$ atm. 2- $2 \mathrm{H}_{2} \mathrm{O} \leftrightarrows \mathrm{H}_{3} \mathrm{O}^{+}+\mathrm{OH}^{-}$. celente rendimento e sem a presença de catalisador ácido, com um tempo reacional de minutos (comparado com várias horas do procedimento tradicional $)^{30}$. Outro fato importante, é que neste experimental não houve a necessidade de adicionar um agente emulsificante, como na metodologia tradicional o que evidencia que este processo é realmente limpo (Esquema 9). A adição de água à carvona também ocorreu sem necessidade de catalisador e de forma seletiva com obtenção de regiosseletividade Markovinikov, embora em baixa conversão $0^{30}$. Segundo os autores, a matéria-prima não reagida é facilmente reciclada e a reação ocorre em $10 \mathrm{~min}$ (um procedimento de literatura forneceu maior conversão, mas com $48 \mathrm{~h}$ e ácido concentrado) ${ }^{30}$.
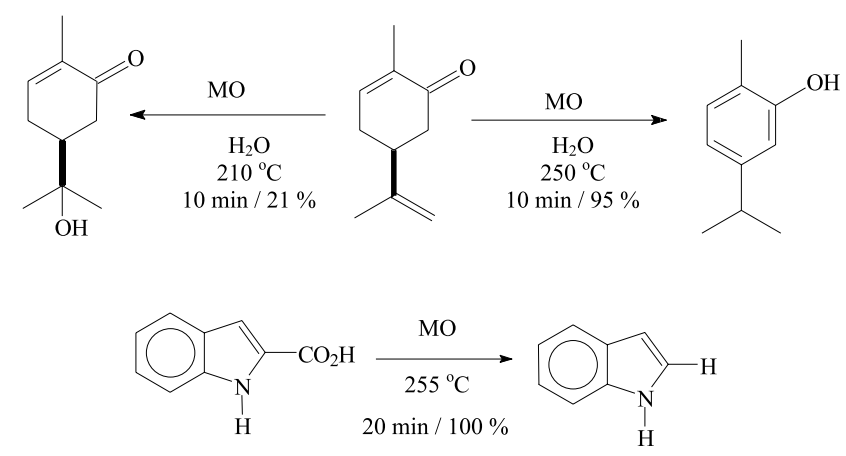

Esquema 9. Reações em água superaquecida com microondas

A descarboxilação do ácido 2-indol-carboxílico em reator de microondas com água superaquecida procede sem a necessidade de um catalisador e com rendimento quantitativo, que segundo Trainor e Strauss ${ }^{30}$ é um método superior às metodologias tradicionais para realizar esta transformação química (Esquema 9).

$\mathrm{O}$ uso de reatores monomodo também já foi apontado como superior ao uso do forno multimodo para radioquímica, pois haveria uma melhor reprodutibilidade, menores tempos de reação (muitos produtos tem uma meia vida curta, como compostos marcados com ${ }^{11} \mathrm{C} t_{1 / 2}=20 \mathrm{~min}$ ), maiores rendimentos e menor formação de rejeitos radioativos ${ }^{33}$. Compostos marcados com ${ }^{3} \mathrm{H},{ }^{11} \mathrm{C} \mathrm{e}{ }^{18} \mathrm{~F}$ possuem grande importância no estudo de processos biológicos e na área de fármacos ${ }^{33}$.

A utilização do aquecimento por microondas é ainda relativamente nova, mas já existe um interesse real em utilizar esta técnica na indústria para síntese orgânica, na destruição de rejeitos orgânicos e na área de processamento de materiais ${ }^{2,34}$. Um exemplo já foi discutido por Stinson, onde um reator de microondas de cloração da companhia Dow Elanco estaria em operação, porém os detalhes deste processo e de outros possíveis processos industriais são escassos ${ }^{34}$.

\section{SELETIVIDADE INDUZIDA POR MICROONDAS}

A sulfonação do naftaleno conduzida em um reator de microondas é considerada como o primeiro caso de seletividade induzida por microondas ${ }^{35}$. Assim, variando-se a potência do aparelho é possível a obtenção seletiva do ácido 2-naftaleno-sulfônico (Figura 3 e Equação 3). É conhecido que a sulfonação do naftaleno com aquecimento convencional fornece o ácido 1-naftaleno-sulfônico a $80{ }^{\circ} \mathrm{C}$ (controle cinético), enquanto que o produto de controle termodinâmico é obtido a > $160{ }^{\circ} \mathrm{C}$ (ácido 2-naftaleno-sulfônico) ${ }^{36}$. O resultado é importante, pois mostra que é possível obter um controle de seletividade com aquecimento por microondas em uma reação química importante, que é tradicionalmente realizada com aquecimento convencional (e, inclusive, discutida em livros de graduação ${ }^{36}$ ). 


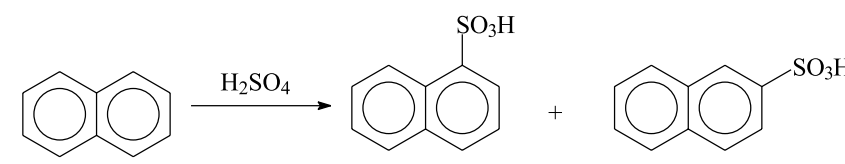

ác. 1-naftaleno-sulfônico ác. 2-naftaleno-sulfônico (ácido 1-NS) (ácido 2-NS)

Equação 3. Sulfonação do naftaleno com microondas

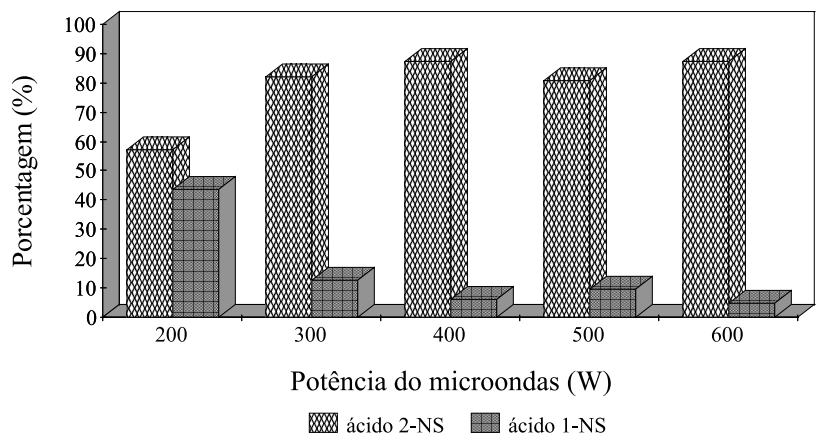

Figura 3. Distribuição do ácido 1-NS e 2-NS com a potência do aparelho

Um exemplo marcante de como o aquecimento por microondas pode levar a um resultado muito diferente do aquecimento convencional foi a resolução enzimática do 1-fenil-etanol catalisada por uma enzima imobilizada ${ }^{37}$ (Equação 4 e Tabela 6). O excesso enantiomérico apresentou um valor bem maior no aquecimento por microondas (93\%) quando comparado com o aquecimento convencional $(62 \%)$ na temperatura de $78^{\circ} \mathrm{C}$. Realmente, ocorreu um maior excesso enantiomérico para o aquecimento por microondas (inclusive em reações de transesterificação catalisadas pela mesma enzima), sendo que a reação foi conduzida em um reator comercial monomodo ${ }^{37}$.

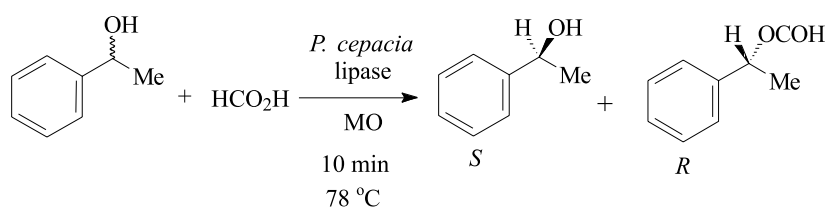

Equação 4. Resolução enzimática com irradiação por microondas

Tabela 6. Resultados da resolução enzimática do 1-fenil-etanol

\begin{tabular}{lccc}
\hline Tipo de Aquecimento & $\begin{array}{c}\mathrm{T} \\
\left({ }^{\circ} \mathrm{C}\right)\end{array}$ & $\begin{array}{c}\text { Conversão } \\
(\%)\end{array}$ & $\begin{array}{c}(S) e e \\
(\%)^{1}\end{array}$ \\
\hline Microondas (10min/30W) & 67 & 45 & 66 \\
Microondas (1min/300W; 4min/80W) & 95 & 47 & 86 \\
Microondas (5 min/60 W; 5min/20W) & 78 & 52 & 93 \\
Convencional (10min) & 78 & 48 & 62 \\
\hline
\end{tabular}

1- determinado por Cromatografia Gasosa de Alta Resolução com coluna quiral.

A benzoilação seletiva com catalisador de estanho de um aminoálcool (Equação 5 e Tabela 7) também apresentou um resultado muito interessante, não apenas por variar a produção de 1 com diferentes potências, mas também por ser um experimento que foi conduzido em um forno de microondas doméstico ${ }^{38}$.

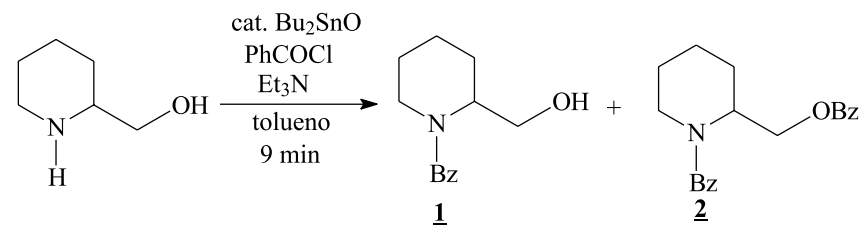

Equação 5. Benzoilação seletiva com aquecimento por microondas

Tabela 7. Resultados da benzoilação seletiva

\begin{tabular}{lcc}
\hline Potência & Rendimento $\underline{\underline{1}}(\%)$ & Rendimento $\underline{2}(\%)$ \\
\hline Máxima & 23 & 12 \\
Média & 21 & 9 \\
Mínima & $\mathbf{8 5}$ & $\mathbf{6}$ \\
\hline
\end{tabular}

\section{EFEITO MICROONDAS}

O chamado "efeito microondas" ou "efeito específico de microondas" tem sido apontado por alguns autores como sendo um efeito não térmico, que seria causado pelo uso da radiação eletromagnética, sendo responsável pelas maiores velocidades de reação com aquecimento por microondas quando comparadas com o aquecimento convencional $^{12,14}$ (diferentes energias de ativação). A expressão, também, tem sido aplicada quando a utilização de microondas leva a um resultado que não foi obtido com o aquecimento convencional, como diferentes seletividades e rendimentos bem maiores.

É um assunto polêmico e de certa controvérsia, já que alguns autores mostraram que determinadas reações possuem a mesma velocidade com microondas ou aquecimento convencional ${ }^{12,14,39,40}$ (como reações de Diels-Alder, ene e outras). A cinética em forno de microondas caseiro é um estudo difícil, já que não há controle de temperatura, e relatos iniciais de reações que foram aceleradas em forno doméstico foram posteriormente analisadas em reatores de microondas onde verificou-se que a velocidade era a mesma se comparada com o aquecimento convencional. Assim, a diferença de velocidade seria explicada em alguns casos por uma medição inadequada da temperatura e pressão ${ }^{12,14,39,40}$. Outro estudo difícil de ser conduzido é a cinética em sistemas heterogêneos, como nas reações sem solvente, onde muitas vezes os reagentes estão suportados numa matriz inorgânica ou são misturados formando uma massa pastosa.

$\mathrm{O}$ aumento de velocidade poderia vir do superaquecimento de solventes orgânicos ${ }^{12,34}$, como a acetonitrila (p.e. $82^{\circ} \mathrm{C}$ ) que quando aquecida por microondas atinge $120^{\circ} \mathrm{C}$ sem ebulição ${ }^{34}$. Este fato, poderia levar a aumentos de 10 a 50 vezes na velocidade da reação $0^{12,34}$.

Ainda faltam estudos mais criteriosos para investigar estes efeitos de seletividade e velocidade, pois é preciso uma melhor compreensão sobre o que realmente ocorre nestes casos. No entanto, é inegável que os fatos experimentais demostram claramente que existem vantagens consideráveis em usar o aquecimento por microondas, como os exemplos selecionados neste trabalho.

\section{CONCLUSÕES}

O aquecimento por microondas é uma forma atraente de conduzir reações orgânicas pela grande redução nos tempos de reação e bons rendimentos. A técnica é promissora do ponto de vista industrial, já existindo vários reatores em uma escala de laboratório.

A utilização de aquecimento por microondas em reações em ausência de solvente tem sido apontada como uma técnica de Química Limpa. 
A possibilidade de controle de seletividades é um campo novo e bastante promissor da química de microondas.

A existência do chamado "efeito microondas" está presente em vários exemplos onde resultados obtidos com irradiação por microondas não são possíveis com aquecimento convencional, porém mais estudos são necessários para uma melhor entendimento deste fenômeno.

\section{AGRADECIMENTOS}

Ao CNPq por uma bolsa de doutorado, e aos professores M. C. S. de Mattos (IQ/UFRJ) e A. L. Gemal (IQ/UFRJ) por discussões e sugestões úteis na disciplina Seminários de Doutorado.

\section{REFERÊNCIAS}

1. Zlotorzynski, A.; Crit. Rev. Anal. Chem. 1995, 25, 43.

2. Dagani, R.; Chem. Eng. News 1997, 75, 26 (10 fevereiro).

3. http://www.raytheon.com/histback.html, acessada em Julho 2001; http:// www.gallawa.com/microtech/history.html, acessada em Julho 2001.

4. Kingston, H. M.; Jassie, L. B., ed.; Introduction to Microwave Sample Preparation, ACS Professional Reference Book: Washington, D.C., 1988.

5. Abramovich, R.; A. Org. Prep. Proced. Int. 1991, 23, 683.

6. Gedye, R.; Smith, F.; Westaway, K.; Ali, H.; Baldisera, L.; Laberge, L.; Rousell, J.; Tetrahedron Lett. 1986, 27, 279.

7. Giguere, R. J.; Bray, T. L.; Duncan, S. M.; Majetich, G.; Tetrahedron Lett. 1986, 27, 4945.

8. Mingos, D. M. P.; Baghurst, D. R.; Chem. Soc. Rev. 1991, 20, 1.

9. Caddick, S.; Tetrahedron 1995, 51, 10403.

10. Bose, A. K.; Banik, B. K.; Lavlisnkaia, N.; Jayaraman, M.; Manhas, M. S.; CHEMTECH 1997, 27, 18.

11. Varma, R. S.; Green Chem. 1999, 1, 43.

12. Strauss, C. R.; Trainor, R. W.; Aust. J. Chem. 1995, 48, 1665.

13. Loupy, A.; Petit, A.; Hamelin, J.; Texier-Boullet, F.; Jacquault, P.; Mathé, D.; Synthesis 1998, 1213

14. Langa, F.; De La Cruz, P.; De La Hoz, A.; Díaz-Ortiz, A.; Díez-Barra, E.; Comtemp. Org. Synth. 1997, 4, 373.

15. Gabriel, C.; Gabriel, S.; Grant, E. H.; Halstead, B. S. J.; Mingos, D. M. P.; Chem. Soc. Rev. 1998, 27, 213.
16. Sanseverino, A. M.; Quim. Nova 2000, 23, 102.

17. Gutierrez, E.; Loupy, A.; Bram, G.; Ruiz-Hitzky, E.; Tetrahedron Lett. 1989, 30,945 .

18. Bram, G.; Loupy, A.; Majdoub, M.; Gutierrez, E.; Ruiz-Hitzky, E.; Tetrahedron 1990, 46, 5167.

19. Spinella, A.; Fortunati, T.; Soriente, A.; Synlett 1997, 93.

20. Khadilkar, B. M.; Bendale, P. M.; Synth. Commun. 1997, 27, 2051.

21. Ranu, B. C.; Saha, M.; Bhar, S.; Synth. Commun. 1997, 27, 621.

22. Sadicoff, B. L.; Amorim, M. C. V.; Mattos, M. C. S. de; Quim. Nova 2000, 23, 557 .

23. Bose, A., K.; Maghar, S. Manhas, M. S.; Ghosh, M.; Shah, M.; Raju, V. S.; Bari, S. S.; Newaz, S. N.; Banik, B. K.; Chaudhary, A. G.; Barakat, K. J.; J. Org. Chem. 1991, 56, 6968.

24. Ram, S.; Ehrenkaufer, R. E.; Synthesis 1988, 91.

25. Bose, A. K.; Banik, B. K.; Barakat, K. J.; Manhas, M. S.; Synlett 1993, 575 .

26. Banik, B. K.; Barakat, K. J.; Wagle, D. R.; Manhas, M. S.; Bose, A. K.; J. Org. Chem. 1999, 64, 5746.

27. Baghurst, D. R.; Mingos, D. M. P.; J. Chem. Soc. Dalton Trans. 1992, 1151; Pagnotta, M.; Nolan, A.; Kim, L.; J. Chem. Ed. 1992, 69, 599; Pecoraro, É.; Davolos, M.; Jafelicci, M. J.; Quim. Nova 1997, 20, 89.

28. Raner, K. D.; Strauss, C. R.; Trainor, R. W.; Thorn, J. S.; J. Org. Chem. 1995, 60, 2456.

29. Loupy, A.; Pigeon, P.; Ramdani, M.; Jacquault, P.; Synth. Commun. 1994, 24, 159.

30. An, J.; Bagnell, L.; Cablewski, T.; Strauss, C. R.; Trainor, R. W.; J. Org. Chem. 1997, 62, 2505.

31. Zurer, P.; Chem. Eng. News 2000, 78, 26 (3 janeiro).

32. Jones, J. J.; Silva, F. M. da; Quim. Nova 2001, 24, 646.

33. Elander, N.; Jones, J. R.; Lu, S.-Y.; Stone-Elander, S.; Chem. Soc. Rev. 2000, 29, 239.

34. Stinson, S;. Chem. Eng. News 1996, 74, 45 (20 maio).

35. Stuerga, D.; Gonon, K.; Lallemant, M.; Tetrahedron 1993, 49, 6229.

36. Morrison, R. T.; Boyd, R. N.; Química Orgânica, $9^{a}$ ed., Fundação Calouste Gulbenkian: Lisboa, 1990, p. 1474-1475.

37. Carrillo-Munoz, J.-R.; Bouvet, D.; Guibé-Jampel, E.; Loupy, A.; Petiti, A.; J. Org. Chem. 1996, 61, 7746.

38. Herradón, B.; Morcuente, A.; Valverde, S.; Synlett 1995, 455; Morcuende, A.; Ors, M.; Valverde, S.; Herradón, B.; J. Org. Chem. 1996, 61, 5264.

39. Laurent, R.; Laporterie, A.; Dubac, J.; Berlan, J.; Lefeuvre, S.; Audhuy, M.; J. Org. Chem. 1992, 57, 7099.

40. Stadler, A.; Kappe, C. O.; J. Chem. Soc., Perkin Trans. 2, 2000, 1363. 